\title{
Friedel-like oscillations in carbon nanotube quantum dots
}

\author{
A. Ayuela, ${ }^{1, a)}$ W. Jaskólski, ${ }^{2}$ M. Pelc, ${ }^{2}$ H. Santos, ${ }^{3,4}$ and L. Chico ${ }^{4}$ \\ ${ }^{1}$ Donostia International Physics Center (DIPC) and Unidad de Física de Materiales, Centro Mixto CSIC- \\ UPV/EHU, 20080 Donostia, Spain \\ ${ }^{2}$ Instytut Fizyki UMK, Grudziadzka 5, 87-100 Toruń, Poland \\ ${ }^{3}$ Departamento de Física Aplicada, Facultad de Ciencias del Medio Ambiente, Universidad de Castilla-La \\ Mancha, 45071 Toledo, Spain \\ ${ }^{4}$ Instituto de Ciencia de Materiales de Madrid, Consejo Superior de Investigaciones Científicas, C/Sor \\ Juana Inés de la Cruz 3, Cantoblanco, 28049 Madrid, Spain
}

(Received 9 June 2008; accepted 4 September 2008; published online 30 September 2008)

\begin{abstract}
The interface states of all-metallic carbon nanotube quantum dots are studied based on a tight-binding approach and a Green's function matching technique. We have found that depending on the type of metallic tube, the energy of interface states may show an oscillatory behavior. We identify these as steamming from Friedel oscillations. We comment on the possible implications of this finding on other physical properties, such as stability during the growth of nanotube junctions and magnetic interaction through carbon nanotubes. (C) 2008 American Institute of Physics. [DOI: $10.1063 / 1.2988644]$
\end{abstract}

Recent technological advances in the production and processing of carbon nanotubes (CNTs) allow for the fabrication of electronic devices, such as diodes, ${ }^{1,2}$ transistors, ${ }^{3}$ and even an entirely CNT-based radio. ${ }^{4}$ However, further progress depends on the ability to form junctions between different tubes. Quite recently, the controlled synthesis of several CNT intramolecular junctions by temperature changes during growth ${ }^{5}$ was reported. This finding may allow for the controlled production of CNT quantum dots (QDs) and superlattices (SLs), providing more complex single-wall carbon tube nanoelectronic components. Notwithstanding the remarkable success in fabricating these junctions, the electronic structure of interface topological defects is not fully understood. ${ }^{6-10}$ It is one of the main topics of this letter to study these defect-related states and their coupling through the metallic QD.

Quantum modulations are widely investigated for different nanostructures-atomic clusters, ${ }^{11}$ nanowires, ${ }^{12}$ layers, ${ }^{13-16}$ and multilayers. ${ }^{17-20}$ Much less effort has been devoted so far to the study of quantum oscillations in CNTs. Ferreira et $a .^{21}{ }^{21}$ investigated local electronic properties of CNT heterojunctions, but the nature of Friedel oscillations in such systems has not been fully clarified. In this letter we intend to shed some light on the nature of such oscillations in CNTs by investigating QDs made of all-metallic tubes. We show that the coupling between interface states depends not only on the length of the QD, but also on the type of tube forming the QD. We find that in some cases the coupling has an oscillatory character for increasing QD length, identifying this feature as resulting from Friedel-like oscillations.

We have performed calculations in order to characterize the energy spectra of $N(12,0)$ and $N(6,6)$ QDs. An $N(12,0)$ QD is made by sandwiching $N$ unit cells of a $(12,0)$ tube between two semi-infinite $(6,6)$ leads, whereas an $N(6,6)$ dot consists of $N$ unit cells of a $(6,6)$ tube between two $(12,0)$ leads, as shown in the upper insets in Figs. 1(a) and 1(b). The junctions between such tubes are made of six pairs of

\footnotetext{
a) Author to whom correspondence should be addressed. Electronic mail: swxayfea@sw.ehu.es.
}

pentagon/heptagon defects. All calculations have been performed within the $\pi$-electron tight-binding approximation. ${ }^{22}$ We have recently shown ${ }^{23}$ that this approximation yields the electronic structure of $N(12,0) / M(6,6)$ SLs around the Fermi energy $E_{F}$ : in good agreement with the results from first-principles calculations. In the investigated systems, localized states appear either due to confinement in the QD, i.e., quantization of some bands of the QD-forming tube, or due to the presence of pentagon-heptagon interface defects. ${ }^{24}$ To account for the discrete energy levels of QD systems, we have calculated the local density of states using the Green's function matching method. ${ }^{25}$

For a ring of pentagon-heptagon defects between two CNTs, the hybridization of $p_{z}$ orbitals yields charge accumulation at the interface. This may produce the appearance of defect states close to the $E_{F}$. This charge is screened by charge density oscillations (Friedel oscillations) in the CNTs. The interface charge accumulation and density oscillations are also well seen in related systems, such as CNT SLs. The charge density Friedel oscillations have their counterpart in the interaction between a pair of charged impurities (see, e.g., Refs. 26-28). In such a case the oscillating charge densities overlap in the region between the interface defects, and because of the interference effects the interaction energy between two interface defects takes the same oscillatory dependence as in the single defect case. This interaction energy is also called Friedel-like. As the energy has a term that is the sum of eigenvalues, to look for Friedel oscillations we focus on the interface states, which, as defect states, give rise to these oscillations.

Since interface states are localized on both junctions, at opposite sides of the QD, one should expect that their energies get closer for increasing QD lengths, i.e., for increasing separation between interfaces. However, Fig. 1 shows that this is not always the case. In Figs. 1(a) and 1(b) we display the energy positions of the interface states versus $N$ for $N(12,0)$ and $N(6,6)$ dots, respectively. The energies of the interface states of $N(12,0)$ dots decrease monotonically as expected, while the energies of the interface states of $N(6,6)$ dots show clear oscillations. The amplitude of the oscilla- 

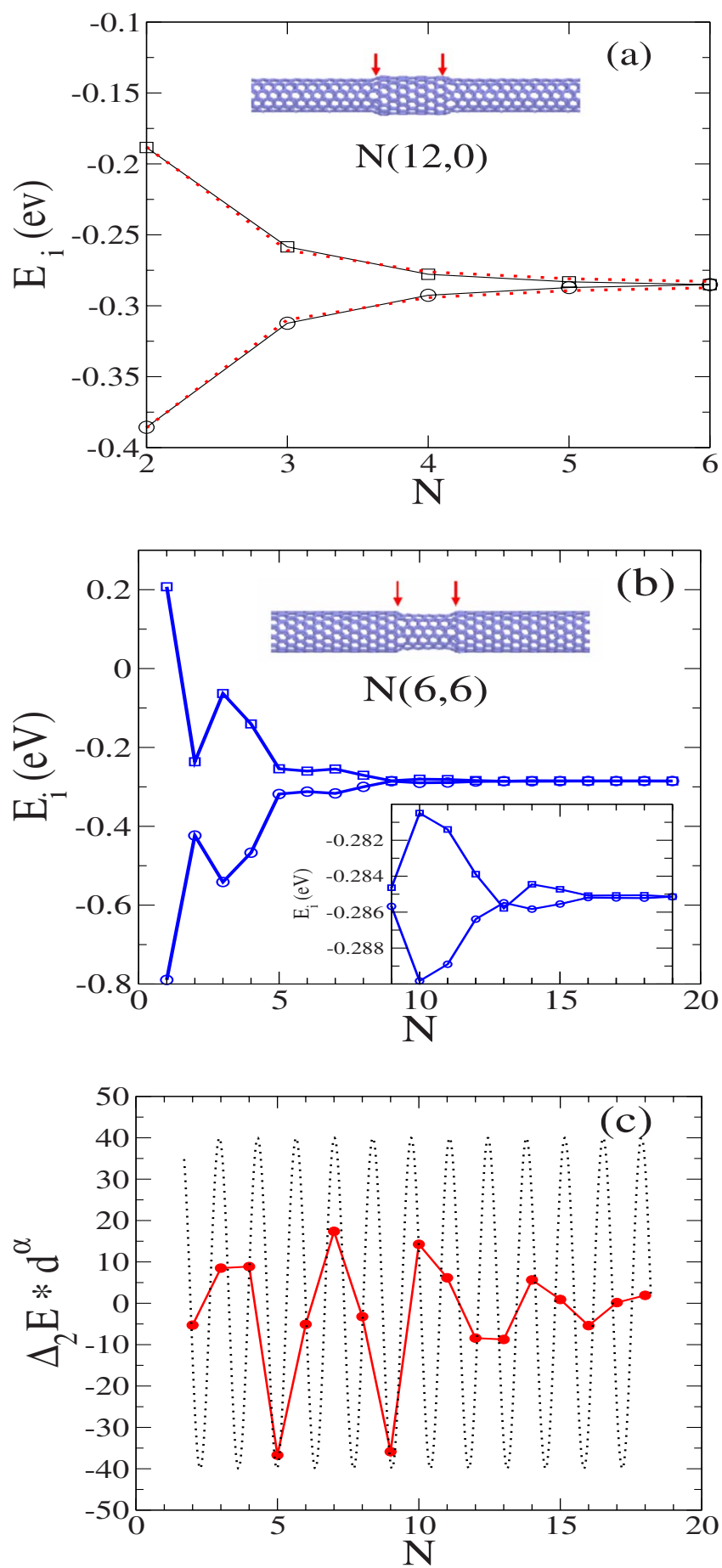

FIG. 1. (Color online) (a) Energies of the interface states of $(6,6) / N(12,0) /(6,6)$ QD vs dot size $N$. (b) The same as (a) but for $(12,0) / N(6,6) /(12,0)$ QD. The upper insets show schematically the QDs, with arrows marking the interfaces. The lower inset in (b) is a zoom in the region of large $N$. (c) Full line with circles: second derivative of the lowest interface state energy for the $(12,0) / N(6,6) /(12,0)$ dot multiplied by the dot size $d^{\alpha}$, and $\alpha$ is the decay exponent extracted from the data shown in (a). Dotted line: fitted function $f(d) d^{\alpha}$, where $f(d)$ is defined by Eq. (1).

tions increases with decreasing $N$. Below, the observed effect is explained based on the coupling of the dot-lead interface states via Friedel-like charge oscillations inside the QD.

In both kinds of QDs, the decay and oscillations of the energies of interface states should implicitly depend on the QD length $d$ and on the Fermi wavevector $k_{F}$ of the metallic inner tube, which constitutes the dot. This explains the different behaviors of the two QD series: for the $(12,0)$ nano- tube, we have $k_{F}=0$, while for the $(6,6)$ tube, we have $k_{F}$ $\sim 2 \pi / 3 a$, where $a=2.46 \AA$ is the length of the unit cell of any $(n, n)$ nanotube. Therefore, the $(12,0)$ QD series cannot show any oscillations in the coupling of interface states via the inner zigzag nanotube dot. In contrast, the coupling of interface states via the $(6,6)$ QD could reveal some oscillations related to the nonzero value of the Fermi wavevector.

To study the observed oscillations and to find the decay rate for the energies of localized interface states, we focus on one of them, e.g., the lowest one, and we investigate its energy dependence versus the system size. Due to the decreasing amplitude of the oscillations for large distances, it is customary to focus on the second derivative of the magnitude of interest, where the oscillations are clearer. ${ }^{15,16,29-32}$ We have calculated the aforementioned derivative of the energy versus QD thickness (i.e., versus the number of unit cells, $N$ ) and have fitted the results to the function that governs Friedel-like oscillations,

$$
f(d)=\frac{A \sin \left[2 k_{F}\left(d+\delta_{0}\right)\right]}{d^{\alpha}},
$$

where $d$ is the QD length and $\delta_{0}$ is a surface shift that accounts for the wavefunction spill out of the dot edges. In Fig. 1 (c) we show our results for the $N(6,6)$ dots plotted with a solid line. Our calculations are in excellent agreement with Eq. (1) (dotted lines in Fig. 1).

The damping factor $\alpha$ can be directly extracted from the results for the $N(12,0)$ QD presented in Fig. 1(a) since in this case $k_{F}=0$, and there are no oscillations. Surprisingly, we find that the $\alpha$ exponent is around 3.5, instead of the expected value for a one-dimensional system, which should be $\alpha \approx 1{ }^{33}$ Therefore, we have found that the coupling of the interface heptagon-pentagon defects in CNT dots of small diameter does not follow the damping of one-dimensional systems.

With the damping factor obtained for the interface states of the $N(12,0) \mathrm{QD}$, we can proceed to study the oscillations of the interface levels of the $N(6,6)$ dot. The interference between the two characteristic lengths, unit cell size $a$ $=d / N$ and $\left(2 k_{F}\right)^{-1}$, gives the impression of a longer periodicity, the so-called "aliasing" effect. We have fitted the oscillation period to 1.4 unit cells, very close to the theoretical value, $\frac{3}{2}$, obtained from $k_{F}=(2 / 3)(\pi / a)$. Hence, we conclude that the analytical expression [Eq. (1)] due to Friedel oscillations gives a very good approximation to the coupling of interface states through metallic carbon tubes. We have checked that the same value is obtained for other $(2 n, 0) / N(n, n) /(2 n, 0)$ QDs.

These results can be generalized to other CNT systems. We have performed calculations for interface bands of $M(12,0) / N(6,6)$ SLs, finding that Eq. (1) is also valid, with a period of 1.53 unit cells, even closer to the theoretical value.

The appearance of Friedel-like oscillations in CNT-based systems is not restricted to interface energy levels. This oscillatory behavior, related to the Fermi wavevector of the metallic constituent, can be expected in other phenomena, which could be experimentally observed. Firstly, if a QD is formed using a nanotube with $k_{F} \neq 0$, the total energy of the system also oscillates with the dot size, so there should be a size selection in the growing process. The total energy minima are associated with the most stable sizes, as already 
observed in layer growth, ${ }^{29,30}$ wires, and clusters. ${ }^{34}$ This will certainly give some sort of "magic numbers" also in CNT dot lengths.

Besides, we would like to remark that the Fermi surface nesting will also influence the magnetic coupling through some metallic nanotubes, as it happens in magnetic multilayers. ${ }^{31,32}$ If a certain amount of charge flows to the QD nanotube, ${ }^{35}$ we expect to have a spin-polarized charge transfer with an oscillatory behavior. Therefore, further experimental work is encouraged in order to identify such oscillatory patterns.

Finally, as shown in Ref. 23, the interface states of the $(2 n, 0) /(n, n)$ SL appear usually just below and above the Fermi level. Thus, the reported oscillatory changes of the separation energy between these states should also be seen in optical experiments.

In summary, we have found that dot-lead interface states in pure CNT systems may show Friedel-like oscillations in the energy of the interface states and the total energy of the system. Oscillations occur only when the QD-forming tube has $k_{F} \neq 0$; otherwise a monotonic behavior is observed. We expect this interaction to be important for the understanding of other physical properties and processes, such as selective dot growth and magnetic interaction of transition metal contacts through CNTs.

We acknowledge financial support by the ETORTEK (NANOMAT) program of the Basque government, the Intramural Special Project (Ref. No. 2006601242), the Spanish Ministerio de Ciencia y Tecnología (Grants No. Fis 200766711-C02-C01 and MAT2006-06242) and the European Network of Excellence NANOQUANTA (NM4-CT-2004500198).

${ }^{1}$ P. G. Collins, A. Zettl, H. Bando, A. Thess, and R. E. Smalley, Science 278, 100 (1997).

${ }^{2}$ J. U. Lee, P. P. Gipp, and G. M. Heller, Appl. Phys. Lett. 85, 145 (2004).

${ }^{3}$ S. J. Tans, A. R. M. Verschueren, and C. Dekker, Nature (London) 393, 49 (1998).

${ }^{4}$ K. Jensen, J. Welden, H. Garcia, and A. Zettl, Nano Lett. 7, 3508 (2007).

${ }^{5}$ Y. Yao, Q. Li, J. Zhang, R. Liu, L. Jiao, Y. T. Zhu, and Z. Liu, Nat. Mater. 6, 283 (2007).

${ }^{6}$ L. Chico, V. H. Crespi, L. X. Benedict, S. G. Louie, and M. L. Cohen, Phys. Rev. Lett. 76, 971 (1996); L. Chico, L. X. Benedict, S. G. Louie, and M. L. Cohen, Phys. Rev. B 54, 2600 (1996); R. Saito, G. Dresselhaus, and M. S. Dresselhaus, ibid. 53, 2044 (1996); J. C. Charlier, Ph. Lambin, and T. W. Ebbesen, ibid. 53, 11108 (1996).

${ }^{7}$ L. Chico, M. P. López Sancho, and M. C. Muñoz, Phys. Rev. Lett. 81, 1278 (1998).

${ }^{8}$ C. G. Rocha, T. G. Dargam, and A. Latgé, Phys. Rev. B 65, 165431 (2002); W. Zhang, W. Lu, and E. G. Wang, ibid. 72, 075438 (2005).

${ }^{9}$ E. Jódar, A. Pérez-Garrido, and A. Díaz-Sánchez, Phys. Rev. B 73, 205403 (2006).

${ }^{10}$ L. Chico and W. Jaskólski, Phys. Rev. B 69, 085406 (2004).

${ }^{11}$ O. Genzken and M. Brack, Phys. Rev. Lett. 67, 3286 (1991).

${ }^{12}$ A. I. Yanson, I. K. Yanson, and J. van Ruitenbeek, Nature (London) 400 , 144 (1999).

${ }^{13}$ B. J. Hinch, C. Koziol, J. P. Toennies, and G. Zhang, Europhys. Lett. 10 341 (1989).

${ }^{14}$ F. K. Schulte, Surf. Sci. 55, 427 (1976).

${ }^{15}$ E. Ogando, N. Zabala, E. V. Chulkov, and M. J. Puska, Phys. Rev. B 69, 153410 (2004); 71, 205401 (2005)

${ }^{16}$ A. Ayuela, E. Ogando, and N. Zabala, Phys. Rev. B 75, 153403 (2007).

${ }^{17}$ S. S. P. Parkin, R. Bhadra, and K. P. Roche, Phys. Rev. Lett. 66, 2152 (1991).

${ }^{18}$ M. T. Johnson, S. T. Purcell, N. W. E. McGee, R. Coehoorn, J. Van de Stegge, and W. Hoving, Phys. Rev. Lett. 68, 2688 (1992).

${ }^{19}$ P. Bruno and C. Chappert, Phys. Rev. Lett. 67, 1602 (1991).

${ }^{20}$ D. M. Edwards, J. Mathon, R. B. Muniz, and M. S. Phan, Phys. Rev. Lett. 67, 493 (1991).

${ }^{21}$ M. S. Ferreira, T. G. Dargam, R. B. Muniz, and A. Latgé, Phys. Rev. B 62, 16040 (2000).

${ }^{22}$ With one $\pi$ orbital per atom, the hopping parameter for nearest neighbors is fixed to $V_{p p \pi}=-2.66 \mathrm{eV}$.

${ }^{23}$ A. Ayuela, L. Chico, and W. Jaskólski, Phys. Rev. B 77, 085435 (2008).

${ }^{24}$ W. Jaskólski and L. Chico, Phys. Rev. B 71, 155305 (2005).

${ }^{25} \mathrm{~F}$. García-Moliner and V. R. Velasco, Theory of Single and Multiple Interfaces (Singapore, World Scientific, 1992).

${ }^{26}$ A. Ayuela and N. H. March, Phase Transitions 81, 387 (2008).

${ }^{27}$ F. Flores, N. H. March, Y. Ohmura, and A. M. Stoneham, J. Phys. Chem. Solids 40, 531 (1979).

${ }^{28}$ K. H. Lau and W. Kohn, Surf. Sci. 79, 623 (1979).

${ }^{29}$ M. H. Upton, C. M. Wei, M. Y. Chan, T. Miller, and T. C. Chiang, Phys Rev. B 93, 026802 (2004).

${ }^{30}$ E. Ogando, N. Zabala, E. V. Chulkov, and M. J. Puska, Phys. Rev. B 69, 153410 (2004).

${ }^{31}$ P. Bruno, J. Phys.: Condens. Matter 11, 9403 (1999).

${ }^{32}$ E. Holmström, A. Bergman, L. Nordström, I. A. Abrikosov, S. B. Dugdale, and B. L. Györffy, Phys. Rev. B 70, 64408 (2004).

${ }^{33}$ R. Egger and H. Grabert, Phys. Rev. Lett. 75, 3505 (1995).

${ }^{34}$ J. A. Alonso and M. J. Lopez, J. Cluster Sci. 14, 31 (2003).

${ }^{35}$ The QD Fermi level is fixed by the metallic leads, that is, at $0 \mathrm{eV}$ within a $\pi$-orbital model. Thus, the dot can get some extra charge from the leads. 